\title{
ON THE ADVANTAGES OF OPENING CERTAIN INTRACRANIAL ANEURYSMS*
}

\author{
BY \\ J. R. GIBBS \\ From the South-east Metropolitan Regional Neurosurgical Centre Brook General Hospital, Woolwich, London
}

Of the various techniques available in the treatment of intracranial aneurysm, none is universally applicable to any particular type of case, and it is unwise for a surgeon to be habitually committed to any one procedure, or to attack an aneurysm with a fixed plan in mind.

Three cases of intracranial aneurysm are reported here, two on the middle cerebral artery and one on the internal carotid artery in the cavernous sinus. They each illustrate some of the indications for opening an aneurysm deliberately.

With regard to the middle cerebral aneurysms, there seem to be three indications for herniotomy: (1) A sac so large and thin walled that it cannot safely be retracted to afford a proper view of its origin; (2) a wide neck to the aneurysm, a quite common finding in middle cerebral cases, and generally speaking the larger the sac the more sessile it is; (3) an anatomical arrangement discovered at the neck likely to endanger the patency of the parent vessel when the neck is ligated or clipped.

Atraumatic proximal occlusion of the middle cerebral artery is an essential preliminary to herniotomy, and it is therefore proposed to describe first the clamp which has been used (Fig. 1).

It has roughly the same proportions as a Cairns clip applicator; in fact, the first model was made by modifying one of these. The long blades are placed across a middle cerebral artery proximal to an aneurysm, and closed sufficiently for the single tooth on the handles to engage. The vessel is then "guarded" but the leaf spring between the handles prevents it from being compressed. The clamp is long and light and easily held steady in the wound by an assistant whose hand is rested on the skull. If it is necessary to occlude the artery, the handles are gently closed and the key inserted to keep them so. I have found this clamp efficient and gentle. It is necessary to cover only one blade with rubber.

Perhaps it may be mentioned here that in all cases

* The substance of a paper read before the Society of British Neurological Surgeons at Bristol, on November 24, 1956. of craniotomy for middle cerebral aneurysm, whether herniotomy is intended or not, such a clamp should if possible be applied to the proximal feeding vessel before the dangerous dissection of the aneurysm is completed; every effort is made to by-pass the aneurysm during the approach, exposing its anterior surface only and merely glimpsing the neck. When once the guarding clamp is applied, much of the anxiety of dissection disappears. I have used this method on several occasions, and now consider it foolhardy to deal with the neck of an aneurysm until the middle cerebral artery has been guarded.

\section{Case Reports}

Case 1.-A previously healthy man aged 31 was found to be dysphasic on recovering consciousness after a subarachnoid haemorrhage on March 8, 1954. On the following day he had a generalized fit in which clonus was chiefly right-sided. Percutaneous angiography discovered a large aneurysm on the left middle cerebral artery (Fig. 2).

Timidity led me on March 23 to ligate the left common carotid artery, after clamping it for $\mathbf{2 0}$ minutes had not affected his now almost normal speech.

One month later (April 28, 1954) an open angiogram proved the aneurysm to be at least as large as before, and immediately craniotomy followed.

At operation the lateral aspect of the aneurysm wall was discovered to be so thin that the turbulence of circulating blood could be seen through it. Identification of the vessels deep to the aneurysm was tedious, but eventually what was thought to be the middle cerebral artery was isolated and the guarding clamp applied across it. When the clamp was closed momentarily, the visible surge of blood ceased, and preparations were made to open the sac. Fortunately it was realized in time that even if the artery thus guarded were distal to the aneurysm, the turbulence would still be stopped by occluding it. The clamp was therefore removed and further search continued until both entering and leaving vessels were exposed, disturbing the aneurysm itself as little as possible. It was then obvious that the artery formerly guarded had been distal to the neck. Whereas no increase in the size of the aneurysm had been observed on clamping the exit vessel, trial closure of the entering 


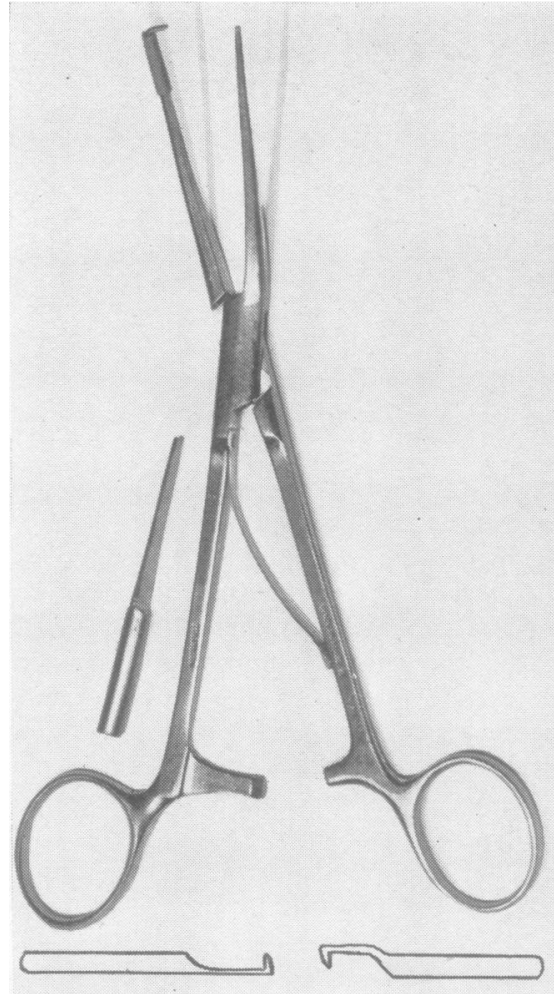

$a$

FIG. 1.-(a) Clamp and key. (b) Clamp guarding.

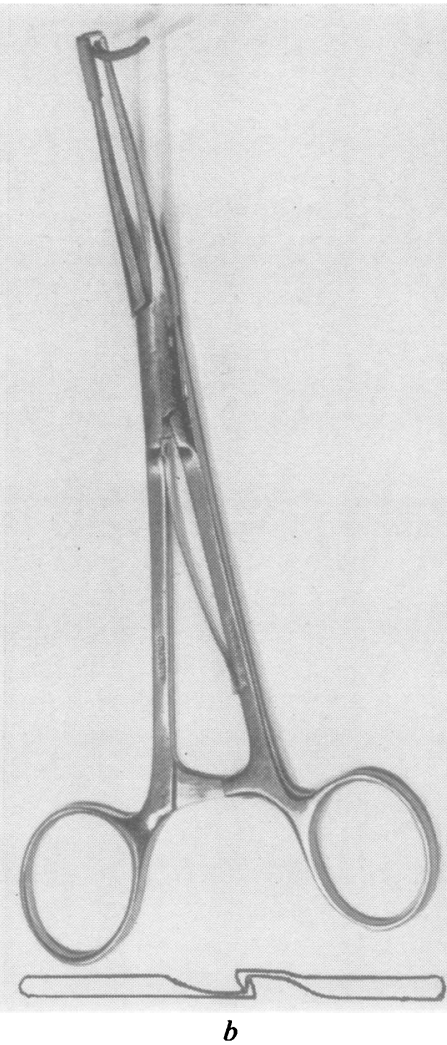

$b$

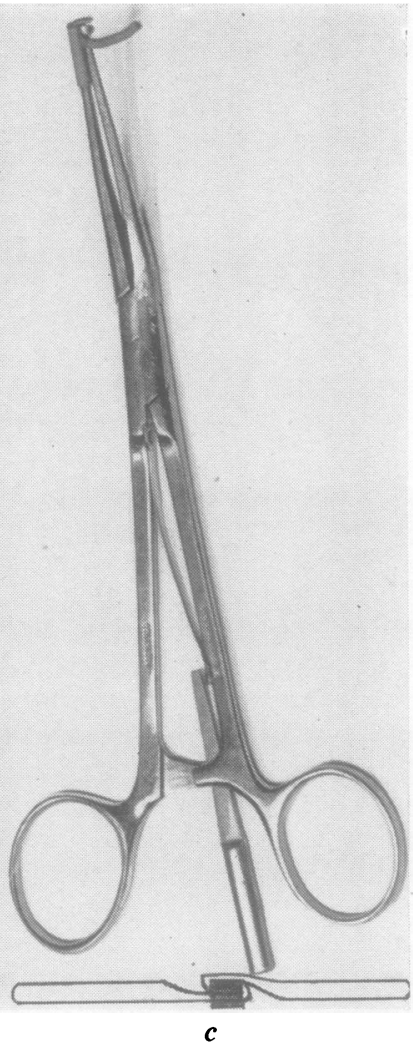

c vessel resulted not only in cessation of the turbulence but slight shrinkage of the sac. After several small

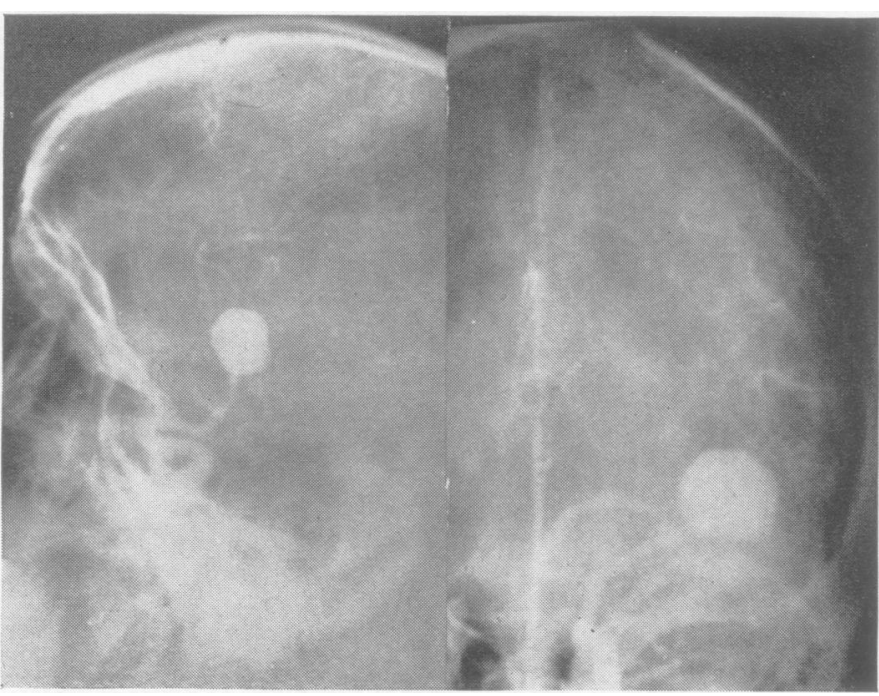

Fig. 2.-Case 1. Pre-operative angiograms. arteries stretched over the aneurysm had been displaced the clamp was closed, the key inserted, and the sac freely opened. The collapsed sac was twisted, held up with forceps by an assistant, and ligated with silk at its neck. At this stage an unexpectedly long time was spent in adjusting the ligature to my liking, and when the key was finally removed from the clamp, it was reported to have been in place for 10 minutes. No hypotensive or freezing techniques were used.

On the first post-operative day the patient was conscious, had no hemiparesis but responded to conversation by grunting only. On the second post-operative day it was evident that he understood what was said to him and said " yes " when asked if he was comfortable. By the time of his discharge three weeks later his speech showed a minor nominal defect only, and it soon returned to normal. He resumed work as a pasteuriser on August 25, and has remained well since apart from a single seizure 11 months after operation. 
Fug. 3.-Case 1. Angiograms one year after craniotomy.

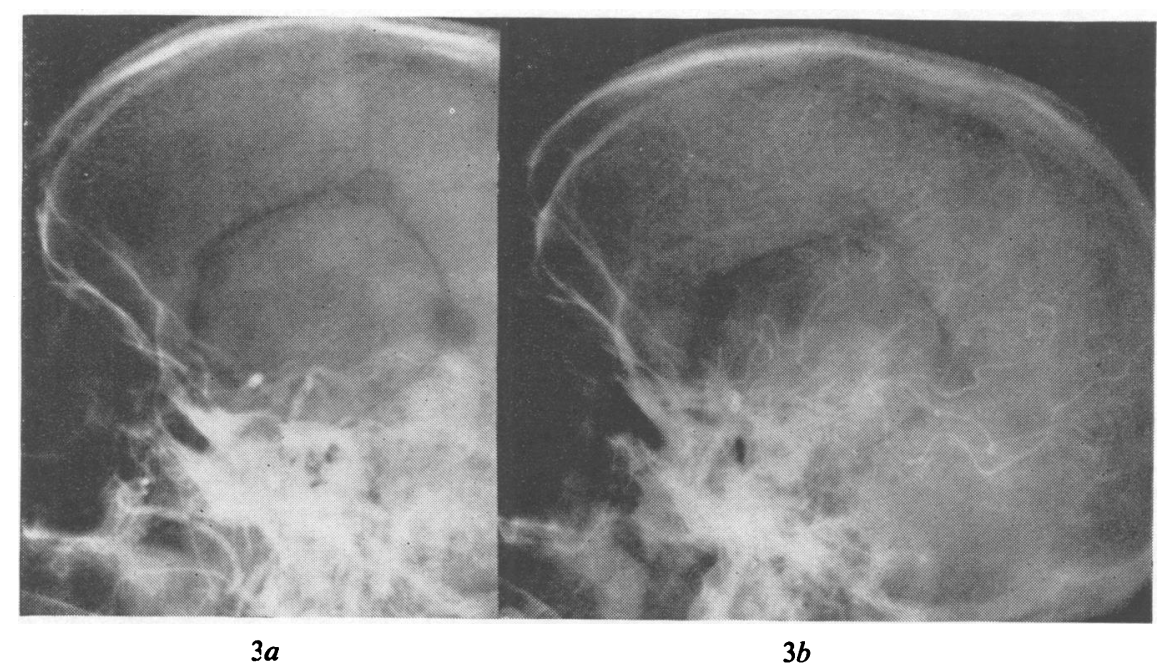

One year after craniotomy (May 3, 1955) he was readmitted for repeat angiography. Under general anaesthesia the left internal carotid artery was exposed and $10 \mathrm{ml}$. of $35 \%$ pyelosil injected into it. The lateral film showed filling of the vertebral and posterior communicating arteries but no contrast in the carotid tree (Fig. 3a); in spite of this there was a small circular shadow approximately in the position of the former aneurysm.

On the grounds that spasm might be responsible for this anomalous filling, the injection was repeated after five minutes, during which time the needle was left undisturbed in the artery. On this occasion the carotid and middle cerebral arteries were demonstrated, but not the vertebral; the small circular shadow was still visible, and will be commented upon later (Fig. 3b). In this connexion it may be relevant to quote from my operation note made after craniotomy. It reads: " Though there is no sac filling now, the fact that there was no neck necessarily means that part of the sac wall will form the wall of the middle cerebral artery over a distance of say $3 \mathrm{~mm}$. However, the wall here was quite thick and I feel will not be a dangerously weak patch ". It remains to be seen whether this optimism was justified. The patient has agreed to undergo repeat angiography five years after operation.

Case 2.-A woman, aged 39, a typist, suffered a subarachnoid haemorrhage on August 29, 1954. The case was submitted for investigation after six weeks bed rest since the haemorrhage, and the blood pressure was then $160 / 110 \mathrm{~mm}$. Hg. There was a previous history of toxaemia of pregnancy.

Angiography discovered a large middle cerebral aneurysm at the common site, on the right side (Fig. 4).

At operation on October 21, 1954, as in Case 1, the Sylvian fissure was opened with the aid of continuous lumbar drainage. Before craniotomy was begun a guarding clamp was placed over the internal carotid artery in the neck, but it was never used. A small
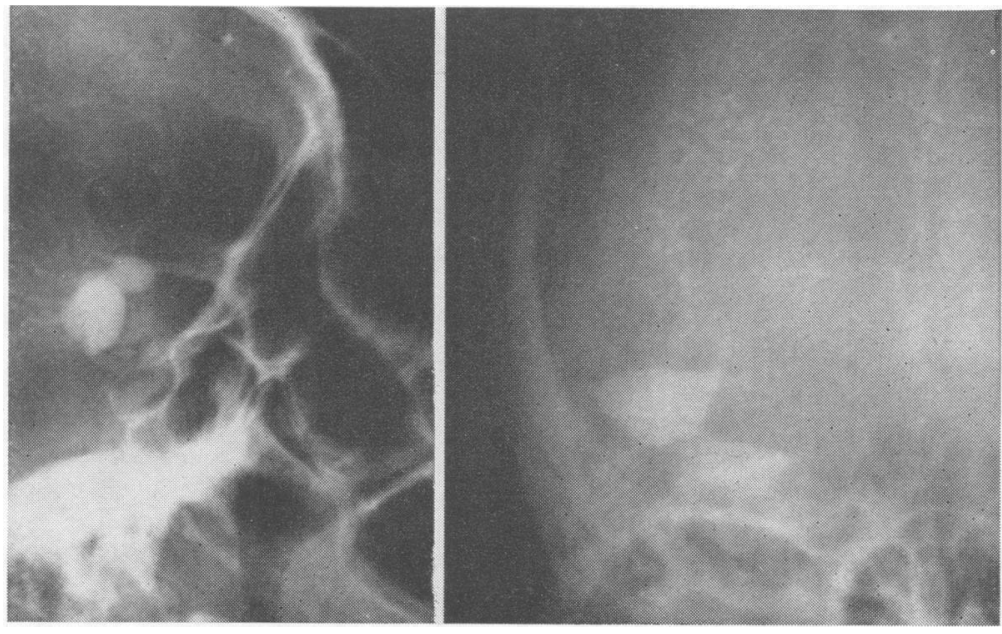

FIG. 4.-Case 2. Pre-operative angiograms. 


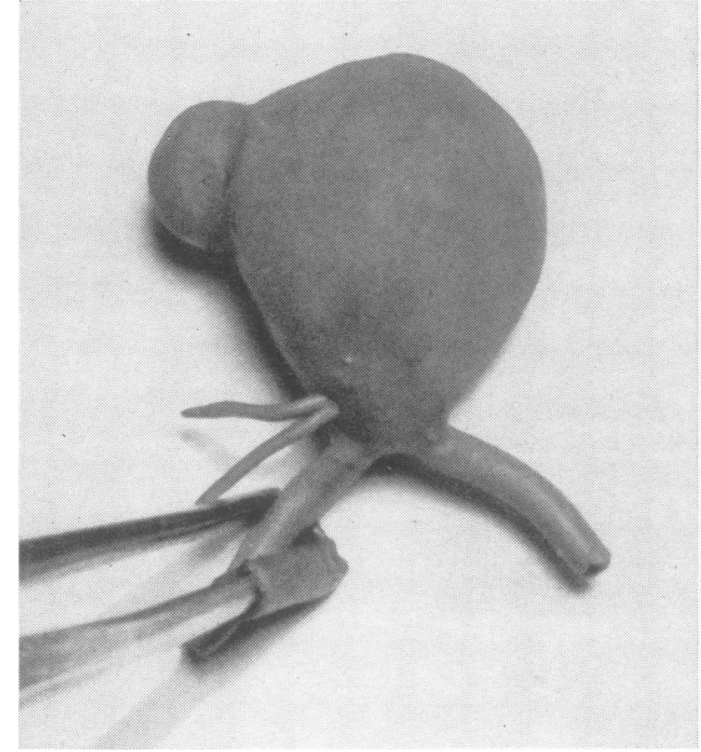

Fig. 5.-Case 2. Guarding clamp applied and dissection of the aneurysm completed. Model from operation sketch.

haemorrhagic cyst in the temporal lobe was evacuated. Again, as in Case 1, the first aim in dissection was to isolate the feeding middle cerebral artery. In spite of induced vascular hypotension to between 80 and $90 \mathrm{~mm}$. of mercury with " arfonad", there were many delays caused by small haemorrhages, not of aneurysmal origin, but after a tedious dissection the feeding

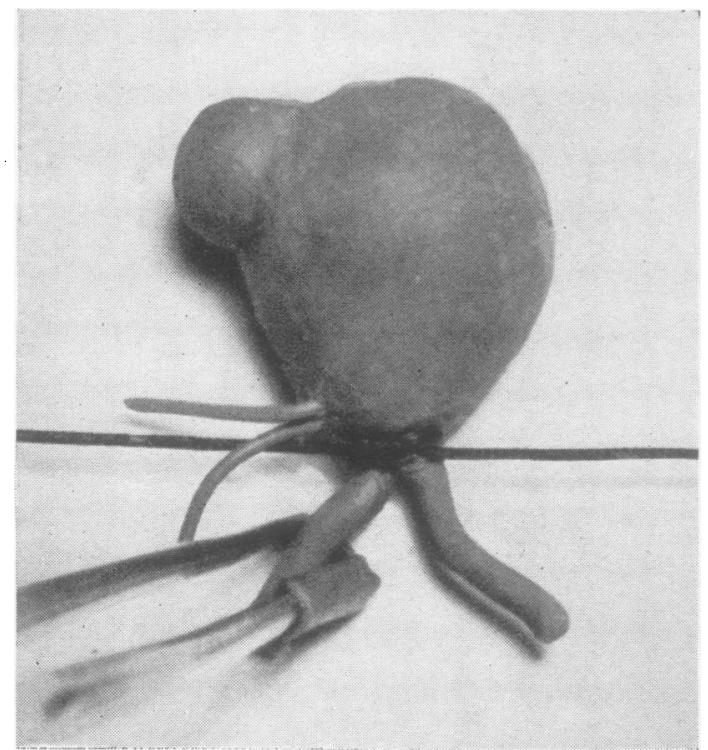

Fig. 6.-Case 2. Occlusion of middle cerebral artery on tightening ligature. A second attempt with the clamp closed had the same result. vessel was straddled by the guarding clamp.

It was then possible to turn attention to the aneurysm itself. It had a transparent fundus and an anterior loculus about $\frac{1}{4}$ in. in diameter, densely adherent to brain and evidently the site of previous rupture. Various small vessels were severed and stripped off the aneurysm sac; and finally only two adventitious ones of moderate size remained, connected with the sac almost at the same spot (Fig. 5).

It was uncertain at this stage whether these were two separate arteries leaving the sac (in which case their function would have to be sacrificed) or whether they were limbs of a single vessel adherent to the sac at an " elbow". At this stage it was thought best not to sever the connexion, for the arteries were of significant size and possibly important.

It was hoped to complete obliteration of the aneurysm without closing the guarding clamp, but when a ligature was placed in position it was quite evident that tightening it would not result in occlusion at the proper place.

Even after inserting the key in the clamp had reduced tension in the aneurysm, the belly of the sac forced the ligature to slide down as it was tightened and so threaten the patency of both entering and exit vessels (Fig. 6).

With the guarding clamp still closed, therefore, the aneurysm was opened widely, part of the incision lopping off the loculus, to save dissecting it. When the lax walls were held up by forceps it was found possible to place the ligature at the optimum level, withoug embarrassing the middle cerebral flow (Fig. 7).

Bleeding from the sac before tightening of the ligature was free, and will be commented upon later, but there was no bleeding from the sac after the ligature was tied proving that the two small arteries mentioned earlie

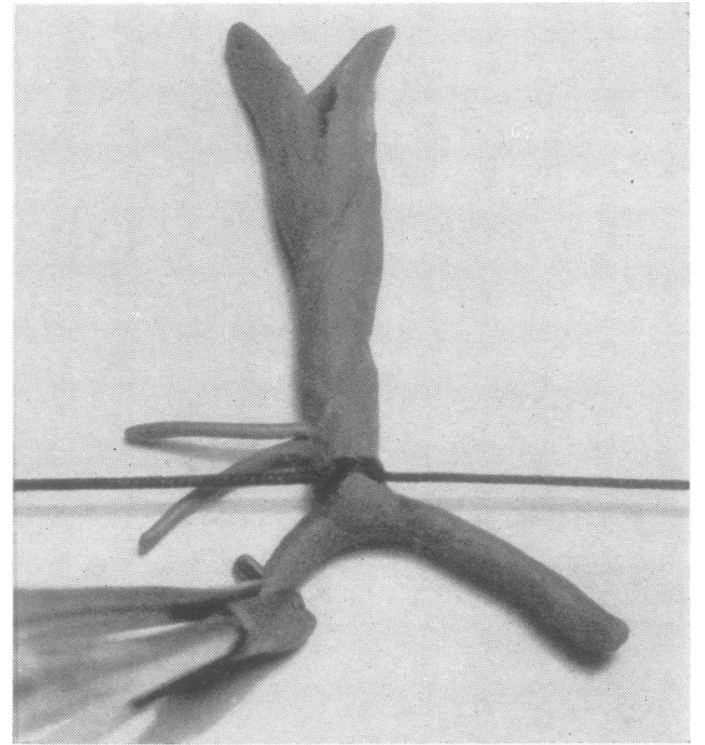

Fig. 7.-Case 2. Correct ligation possible after collapsing the aneurysm. 
were in fact two limbs of a single vessel and had been preserved.

Tightening the knot caused brisk haemorrhage, afterwards proved to be due to damaging a small artery (not shown in the figures) adherent to the aneurysm; it was necessary to use a large sucker to keep the field clear, but bleeding was finally stopped by pressure with haemostatic for a few minutes. When the field was dry the key was removed from the guarding clamp, and it was reported to have been in place for $\mathbf{2 3}$ minutes.

Recovery of consciousness was not delayed, and on the first post-operative day the only weakness discernible was minimal reduction of power in the left grip, not present 24 hours later. Convalescence was uneventful.

One year after craniotomy right percutaneous angiography filled all vessels in the middle cerebral group as before operation, but there was no trace of the aneurysm

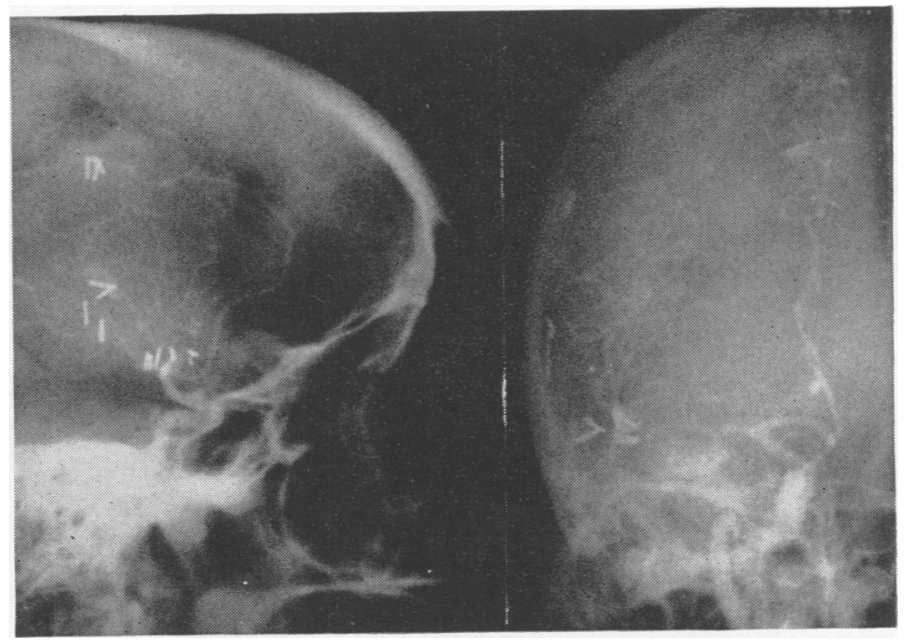

FIG. 8.-Case 2. Angiograms one year after craniotomy.

brisk bleeding from it; the flow must come from the " exit vessel", and is presumably an index of the collateral circulation.

In both cases dissection confirmed the angiographic appearances, in that there was no major branching of the middle cerebral artery at the aneurysm neck. (Trifurcation may make even the most determined surgeon resort to external reinforcement.)

The late angiogram in Case 1 shows a small shadow (Fig. 3) - either a remnant or a recurrence. The latter possibility opens an old question, Does a ligature or a silver clip necessarily leave a weak patch proximal to it which will again enlarge ? As far as Case 1 is concerned, I think a defect of technique was responsible rather than a recurrence. The neck of the sac was several millimetres wide, and I am now of the opinion that a ligature should only be used when the neck of the sac is no wider than the diameter of the parent vessel.

Perhaps we do not open enough cerebral aneurysms; for besides the argument that doing so sometimes makes an otherwise impossible closure feasible, there is the likelihood that some postoperative hemiplegias we have blamed on spasm are really due to manipulating an aneurysm with some clot in it, thus passing a portion of clot on into the distal circulation. Opening an aneurysm after occluding its entering vessel would wash out any friable clot present.

Case 3 presents a different problem, that of severe facial pain due to compression by a non-fistulous aneurysm of the internal carotid artery in the cavernous sinus, not relieved by internal carotid 
ligation. Here, incision of the aneurysm was necessary for therapeutic rather than technical reasons.

Case 3.-A woman aged 51 first complained of double vision in December, 1954, and it lasted eight months. On the morning of December 24, 1955, she awoke with severe pain in the left eye and left side of the forehead. She vomited, and in a few hours the left eye closed.

On admission to the S.E. Metropolitan Regional Neurosurgical Centre two days later she was fully conscious and complaining of headache over the left eye. Ptosis was complete. The only ocular movement possible on that side was in an outward direction, and within two days that also disappeared. Diminished sensibility was found over the first division of the fifth nerve but the corneal reflex was preserved. The pupil was moderately dilated, and fixed. No bruit was audible, and there was no deafness to simple tests. Blood pressure was $180 / 100 \mathrm{~mm}$. Hg. Angiography confirmed the diagnosis of aneurysm in the cavernous sinus, a small shadow showing on the lateral film (Fig. 9).

A plain radiograph of the skull showed widening of the sphenoidal fissure (for what it is worth) and thinning of the greater wing (Fig. 10).

On January 2, 1956, the common carotid artery was ligated without incident, and two days later there was perceptible elevation of the upper lid. By the time of her discharge 10 days afterwards the only other change was slight lessening of the supraorbital pain.

On January 30 her doctor reported that since her discharge she had been suffering the most intense pain

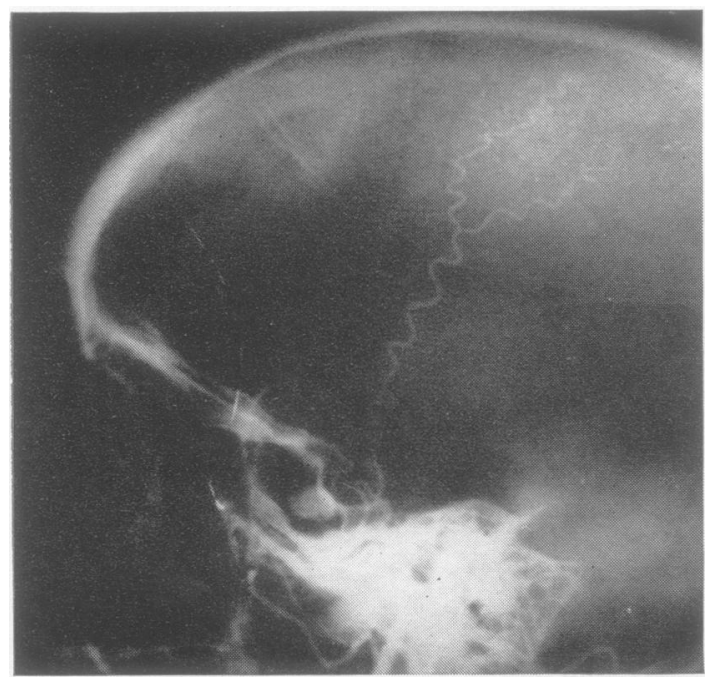

FIG. 9.-Case 3. Pre-operative angiogram.

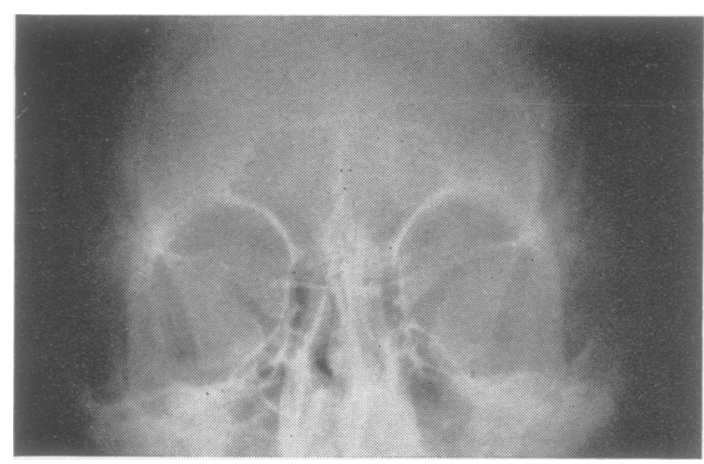

Fig. 10.-Case 3. Widening of the left sphenoidal fissure.

behind the eye and on the left side of the forehead and cheek, and ordinary analgesics proving ineffective, he requested approval of the use of morphia. Readmission was arranged. Ptosis and ophthalmoplegia were then complete, the pupil was still semi-dilated, and the sensory impairment over the fifth nerve territory unchanged except that the corneal reflex was now absent. Blood pressure was $200 / 120 \mathrm{~mm}$. $\mathrm{Hg}$. For the next three weeks the patient lay in bed with her face covered, in great pain while I wondered what to do.

On February 29 an internal carotid angiogram? produced only the faintest of small shadows in thee cavernous sinus, but on the antero-posterior and oblique films the aneurysm appeared outlined by a linear semi circle-presumably a vessel skirting it (Figs. 11a and b) The internal carotid was ligated but this afforded no relief of pain.

On March 6, 1956, 10 weeks after the onset of her: illness and nine weeks after common carotid ligation, craniotomy was undertaken with the intention of evacuating the aneurysm, which was presumed to be tightly distended with clot.

This indeed proved to be the case. At operation three or four adhesions between the under surface of the temporal lobe and the dura of the middle fossa contained small veins, judged at the time to be adventitious venous channels attempting to compensate for the obliteration of the cavernous sinus. About $\frac{1}{3}$ in. of the internal carotid was visible between the aneurysm and the bifurcation; it evidently contained fluid blood, though no pulsation was discernible. It was occluded with a silver clip. No blood escaped through a lumbar puncture needle thrust to a depth of $1 \mathrm{in}$. into the aneurysm, but when it was withdrawn a little thick black blood escaped. A T-shaped incision was made in the aneurysm wall at its summit and the contents sucked out. Black, tarry blood was surrounded by pinkish-grey organizing clot. Evacuation was continued until no soft clot remained and the wall was about $\frac{1}{8}$ in. thick and no further for fear of damaging the nerves lining it. Afterwards the sac collapsed quite well. There was no bleeding. The cavity was filled with saline, left gaping, and the wound closed.

After operation the pupil on that side was fully 


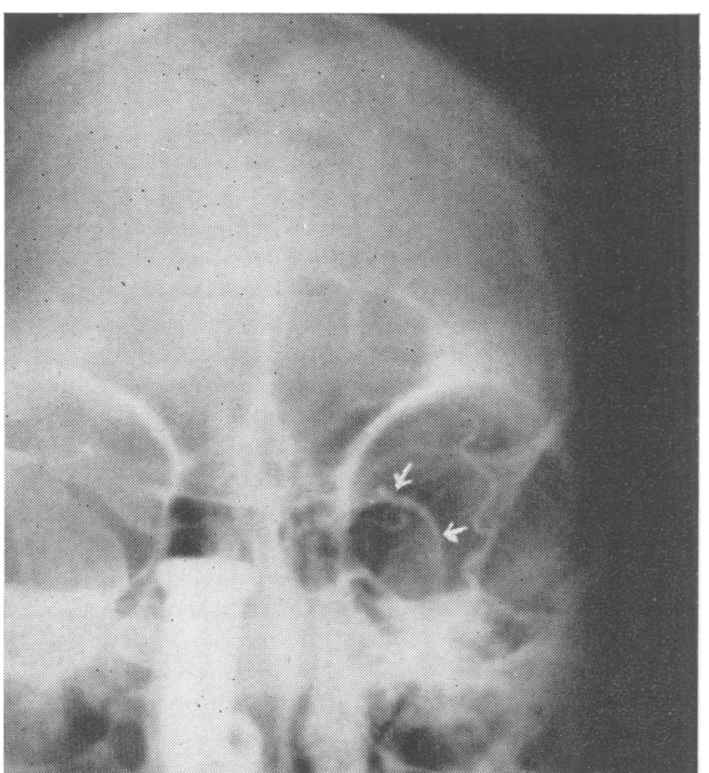

FIG. $11 a$

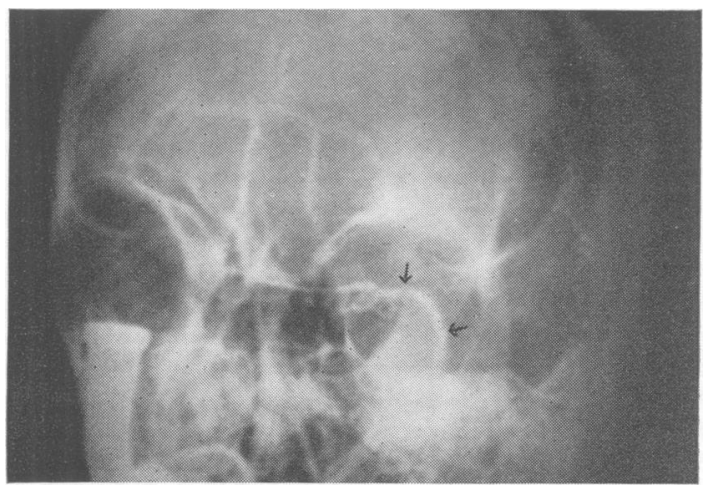

FIG. $11 b$
FIg. 11.-(a) Antero-posterior and (b) oblique angiograms showing vessel skirting the aneurysm sac.*

* I was at a loss to explain this appearance until Mr: Norman Dott identified it as that of an ophthalmic artery arising from the middle meningeal artery - a variation not unknown to him.

dilated and remained so for about 12 hours, when it returned to its former state. The pain was relieved. There was a slight nominal dysphasia.

One year after operation the patient still gets occasional momentary shoots of pain over the forehead-very minor compared with the severe pre-operative pain.

As for the ocular palsies, there has been no appreciable difference in the degree of recovery from that in similar cases treated by carotid ligation or not at all. Abversion and adversion have recovered almost completely, but there is no elevation or depression.

The lid uncovers the pupil, but diplopia confuses her and she prefers to keep the eye closed.

Visual acuity in the affected eye is $6 / 36$, the same as before operation. Sensation over the fifth nerve distribution has improved but is still defective. The corneal reflex has recovered. There is a little excessive tearing. The fundi have remained normal throughout.

Her speech betrays no abnormality in ordinary conversation, but a minor degree of nominal dysphasia still exists; she sometimes cannot name the composer of a piece of familiar music she hears, though recognizing any error in such authorship made by others.

There is slight emotional lability since operation; she cannot endure three hours in a cinema, where drama especially evokes an exaggerated reaction of excitement, and she cries rather too readily at pathos. Her blood pressure is $200 / 110 \mathrm{~mm}$. $\mathrm{Hg}$.

My thanks are offered to Mr. Geoffrey Knight for permission to operate on Case 1, and to Dr. Trevor Griffiths for his very helpful angiographic studies.

The guarding clamp is supplied by Messrs. Lewis Bros. of Marylebone High Street, London. 